\title{
Portosystemic shunt occlusion with balloon-occluded retrograde transvenous obliteration improve refractory hepatic encephalopathy
}

Toru Ishikawa $^{1 *}$, Michitaka Imai ${ }^{1}$, Takashi Owaki ${ }^{1}$, Hiroki Sato ${ }^{1}$, Yujiro Nozawa ${ }^{1}$, Tomoe Sano ${ }^{1}$, Akito Iwanaga ${ }^{1}$, Keiichi Seki ${ }^{1}$, Terasu Honma $^{1}$ and Toshiaki Yoshida ${ }^{1}$

Department of Gastroenterology and Hepatology, Saiseikai Niigata Daini Hospital, Niigata, Japan

A 67-year-old man was referred to us with a diagnosis of hepatic encephalopathy by porto-systemic shunt due to liver cirrhosis hepatitis C infection (Figure 1).

Post admission, oral administration of lactulose and intravenous transfusion of a branced-chain amino acid (BCAA)-enriched solution were performed. However, his venous ammonia level did not decrease significantly. The encephalopathy was refractory to medical management such as lactulose. We decided to perform balloon occluded retrograde obliteration (BRTO) for portosystemic shunt occlusion to prevent hepatic encephalopathy,

Vascular access was achieved using the Seldinger technique. Briefly, an 8-Fr sheath was inserted through the right jugular vein, and a 20 mm diameter balloon catheter (Selecon MP Catheter, Terumo, Tokyo, Japan) was inserted and inflated into the portosystemic shunt (Figure 2). A 2.2-Fr micro-catheter (Progreat, Terumo, Tokyo, Japan) was inserted through the balloon catheter and occlusion by one $22-\mathrm{mm}$ diameter metal coil (IDC; Interlocking Detachable Coil, Boston Scientific, Marlbourough, MA, USA) and seventeen some size fibered metal coils (Interlock-18, Fibered IDC Occlusion System, Boston Scientific, Marlbourough, MA, USA). The most recent venous ammonia level prior to BRTO therapy was $223 \mu \mathrm{g} / \mathrm{dL}$. BRTO with self-expandable coil embolization can achieve satisfactory occlusion of portosystemic shunt and immediate clinical improvement in patients with hepatic encephalopathy. Venous ammonia level post BRTO was $80 \mu \mathrm{g} / \mathrm{dL}$.

Portosystemic shunts may result in chronic portosystemic encephalopathy, a debilitating condition that may be refractory to medical management $[1,2]$. Balloon-occluded retrograde transvenous obliteration (BRTO) is an interventional therapy for the treatment of gastric fundal varices developing in the course of a portosystemic shunt vessel $[3,4]$.

Due to shunt closure by the procedure, BRTO has also been applied to treat portosystemic encephalopathy [4].

In the case of portosystemic encephalopathy, the key factor in pathogenesis is shunting of portal venous blood into the systemic circulation. Thus, shunt closure is a natural interventional target for relief of encephalopathy symptoms. Since its introduction, BRTO has been shown to be a suitable therapeutic option for patients with portosystemic encephalopathy due to various types of extrahepatic portosystemic shunts [5,6]. In conclusion, refractory hepatic encephalopathy to medical management often improve by BRTO.

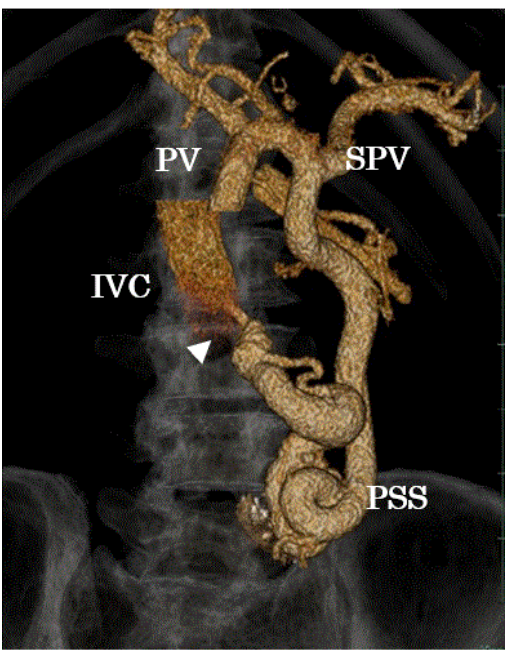

Figure 1. Volume rendering models. A volume rendering models showing a portosystemic shunt from portal vein (PV) to inferior vena cava (IVC). White arrow head shows anastomosis vessel.

PV: portal vein, SPV: splenic vein, IVC: inferior vena cava, PSS: portosystemic shunt.

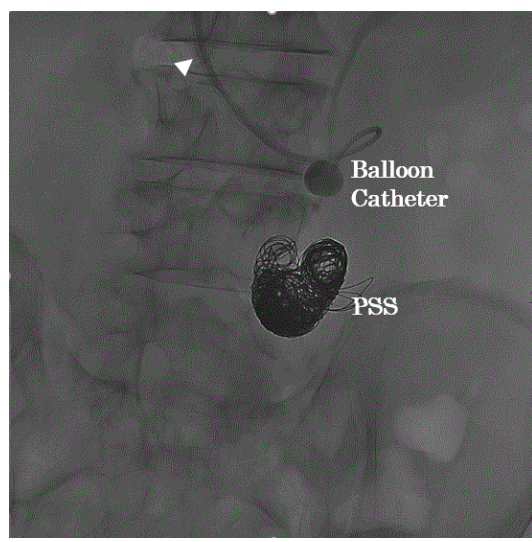

Figure 2. White arrow head shows an 8-Fr sheath through the anastomosis vessel. A 20-mm diameter balloon catheter was inserted and inflated into the portosystemic shunt, and coil embolization was performed to occlude of portosystemic shunt (PSS)

Correspondence to: Toru Ishikawa, MD, Department of Gastroenterology and Hepatology, Saiseikai Niigata Daini Hospital, Teraji 280-7, Niigata 950-1104, Japan, Tel: 81-25-233-6161; Fax: 81-25-233-8880; E-mail: toruishi@ngt.saiseikai.or.jp

Received: August 06, 2017; Accepted: August 16, 2017; Published: August 18, 2017 
Ishikawa T (2017) Portosystemic shunt occlusion with balloon-occluded retrograde transvenous obliteration improve refractory hepatic encephalopathy

\section{References}

1. Uflacker R, Silva Ade O, d'Albuquerque LA, Piske RL, Mourão GS (1987) Chronic portosystemic encephalopathy: embolization of portosystemic shunts. Radiology 165: 721-725. [Crossref]

2. Chandler JG, Fechner R (1983) Hepatopedal flow restoration in patients intolerant of total portal diversion. Ann Surg 197: 574-583. [Crossref]

3. Kanagawa H, Mima S, Kouyama H, Gotoh K, Uchida T, Okuda K (1996) Treatment of gastric fundal varices by balloon occluded retrograde transvenous obliteration. $J$ Gastroenterol Hepatol 22: 51-58. [Crossref]
4. Saad WE, Sabri SS (2011) Balloon-occluded retrograde transvenous obliteration (BRTO): Technical results and outcomes. Semin Intervent Radiol 28: 333-338. [Crossref]

5. Minamiguchi H, Kawai N, Sato M, Ikoma A, Sawa M, et al. (2011) Balloon occlusion retrograde transvenous obliteration for inferior mesenteric vein-systemic shunt. J Vasc Interv Radiol 22: 1039-1044. [Crossref]

6. Tanaka O, Ishihara K, Oyamada H, Harusato A, Yamaguchi T, et al. (2006) Successful portal-systemic shunt occlusion with balloon-occluded retrograde transvenous obliteration for portosystemic encephalopathy without liver cirrhosis. $J$ Vasc Interv Radiol 17: 1951-1955. [Crossref]

Copyright: (C2017 Ishikawa T. This is an open-access article distributed under the terms of the Creative Commons Attribution License, which permits unrestricted use, distribution, and reproduction in any medium, provided the original author and source are credited. 